\title{
Delayed Maturation of the Interstitial Cells of Cajal: A New Diagnosis for Transient Neonatal Pseudoobstruction. Report of Two Cases
}

\author{
By S.E. Kenny, J.M. Vanderwinden, R.J. Rintala, M.G. Connell, D.A. Lloyd, J.J. Vanderhaegen, and M.H. De Laet \\ Brussels, Belgium and Liverpool, England
}

The case histories of two neonates presenting with intestinal pseudoobstruction are presented. One boy infant was premature and the girl infant was full term. Both patients needed a defunctioning ileostomy, and biopsy findings of the intestine in both patients showed a lack of interstitial cells of Cajal (ICC). At the time of closure of the ileostomies to restore intestinal continuity, repeat biopsy results showed a normal pattern of distribution of ICC. Delay in the development of ICC in the gastrointestinal tract may be a cause of intestinal pseudoobstruction in the newborn.

J Pediatr Surg 33:94-98. Copyright 101998 by W.B. Saunders Company.

INDEX WORDS: Neonate, interstitial cells of Cajal, intestinal pseudoobstruction, immunohistochemistry.
G ASTROINTESTINAL OBSTRUCTION in neonates is usually caused by identifiable causes such as mechanical obstruction, meconium ileus, metabolic disturbances and Hirschsprung's disease. A number of patients remain in whom there is a functional obstruction of unknown origin, meconium syndrome, ${ }^{1-3}$ or idiopathic neonatal pseudoobstruction. ${ }^{4}$ Unlike meconium ileus, such syndromes have no association with cystic fibrosis, and its aetiology remains obscure. ${ }^{3}$ A specialised type of cell present in the muscle layers of the gastrointestinal (GI) tract called the interstitial cells of Cajal (ICC), are responsible for the maintenance of GI motility. ICC generate pacemaking slow waves of depolarisation in intestinal smooth muscle and are involved in the coordination of the electromechanical activity of the gut. ${ }^{5-7}$ The tyrosine-kinase receptor c-kit is a specific marker for interstitial cells of Cajal. c-kit and its ligand, the cytokine stem cell factor (SCF), form a trophic system that is required for the development of ICC and the pacemaker component. ${ }^{8}$ Defects of the c-kit/SCF system in mice ${ }^{9,10}$

From the Laboratoire de Neuropathologie, Faculté de Médecine, Department of Pediatric Surgery, Hôpital Universitaire des Enfants Reine Fabiola, Université Libre de Bruxelles, Brussels, Belgium, and the Department of Paediatric Surgery and Institute of Child Health, Alder Hey Children's Hospital and University of Liverpool, Liverpool, England.

The first- and second-listed authors contributed equally to this report.

Presented at the 44th Annual International Congress of the British Association of Paediatric Surgeons, Istanbul, Turkey, July 22-25, 1997.

Address reprint requests to J.M. Vanderwinden, $M D, P h D$, Chercheur Qualifié FNRS, Laboratoire de Neurophysiologie et Physiopathologie du Système Nerveux, Faculté de Médecine, Université Libre de Bruxelles, Campus Erasme, CP 601, 808 route de Lennik, B-1070 Brussels, Belgium.

Copyright 1998 by W.B. Saunders Company

0022-3468/98/3301-0022\$03.00/0 and rats ${ }^{11}$ precludes ICC development, producing a disorganised digestive motility pattern and failure to thrive. In humans, c-kit immunohistochemistry has been used to study the abnormal distribution of ICC in infantile hypertrophic pyloric stenosis, ${ }^{12-14}$ Hirschsprung's disease, ${ }^{15.16}$ and in some cases of hypoganglionosis. ${ }^{17}$ Our hypothesis is that abnormalities of ICC might be involved in some neonates presenting with intestinal pseudoobstruction. We report two infants with neonatal intestinal pseudoobstruction requiring support with an ileostomy and total parenteral nutrition.

\section{CASE REPORTS}

\section{Case 1}

$\Lambda$ boy born at 27 weeks' gestation, (birth weight, $1.2 \mathrm{~kg}$ ), presented at the age of 3 weeks with a 3-day history of abdominal distension and bile-stained gastric aspirates associated with no passage of stool from birth. Plain abdominal radiography findings demonstrated features of small bowel obstruction with a single large loop of air-filled bowel in the right iliac fossa (Fig 1). Contrast enema results showed normalcalibre sigmoid and descending colon, but contrast would not pass the splenic flexure. The infant remained symptomatic after this procedure, and a provisional diagnosis of meconium ileus was made. During laparotomy, a distended segment of distal small bowel containing viscid meconium was resected, and a defunctioning Santulli ileostomy was fashioned. The colon appeared macroscopically normal but there was no peristaltic activity. Biopsy specimens taken from the terminal ileum at the time of operation showed the presence of ganglion cells. The stoma functioned after 7 days, but bile-stained aspirates persisted. Oral feeding was not established for 37 days. Stoma closure was performed at a postconceptual age of 42 weeks (postnatal age, 15 weeks) and postoperative recovery was uneventful. Genetic screening results were negative for cystic fibrosis mutations. The patient is now thriving at the age of 6 months on normal nutrition.

\section{Case 2}

A girl was born at term by a normal delivery. Meconium was passed on the second day of life. The infant was discharged from the hospital, but was readmitted as an emergency at 19 days of life with gross abdominal distension. The child was otherwise asymptomatic. Plain 


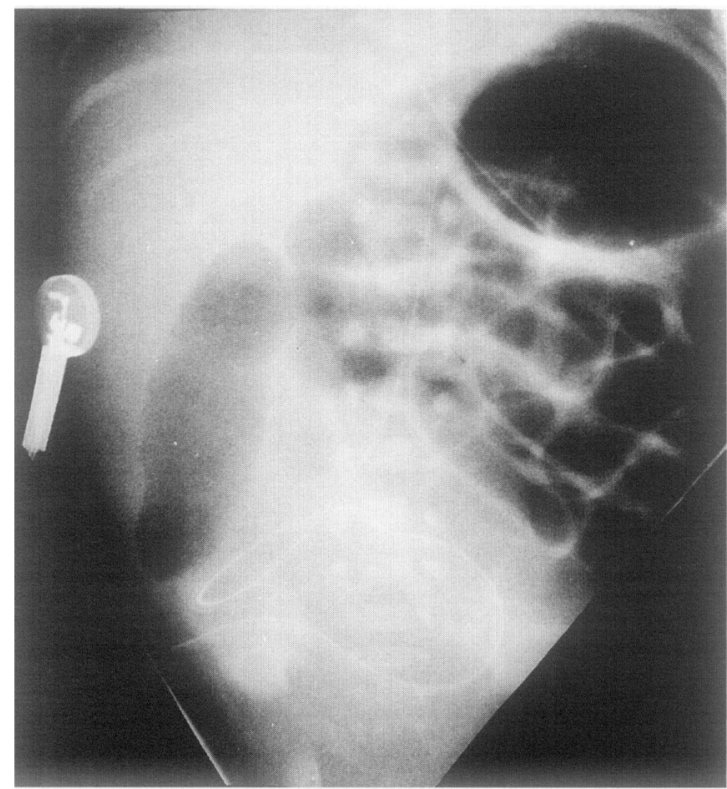

Fig 1. Case 1. Plain abdominal radiograph at 4 days of age shows features of small bowel obstruction with a prominent single loop of gas-filled bowel in the right iliac fossa.

$\mathrm{X}$-ray at the time of admission was suggestive of a distal colonic obstruction (Fig 2A). The diagnosis of Hirschsprung's disease (HD) was suspected. A nasogastric tube was passed and parenteral nutrition commenced. Hydrosoluble contrast enema findings ruled out mechanical obstruction and did not show a transitional zone. Rectal suction biopsies demonstrated submucosal ganglionic cells and the absence of hypertrophic nerve fibres after acetylcholinesterase histochemical staining, thus excluding the diagnosis of HD. Subsequent plain $x$-rays of the abdomen showed an adynamic colon, which retained the contrast medium for 10 days (Fig 2B). The clinical and biological status of the patient remained otherwise normal. At laparotomy performed 12 days after admission ( 1 month of age), the right and transverse colon was dilated, and the diameter of the left colon appeared normal. The entire colon remained completely adynamic during the operation. In contrast, strong propagated contractile activity was noted in the small intestine. Biopsy findings demonstrated the presence of mature ganglion cells at all levels. A defunctioning ileostomy was fashioned, and recovery was uneventful with rapid establishment of oral feeding. At the age of 3 months, a barium enema demonstrated colonic peristalsis with rapid elimination of the contrast medium. Manometry demonstrated a norma recto-anal inhibitory reflex. At 6 months of age the ileostomy was closed and biopsy sections taken of small and large bowels. Recovery was uneventful and the patient is well, with normal bowel habits, after over 30 months of follow-up.

\section{MATERIALS AND METHODS}

\section{Case 1}

At the time of both operations, longitudinal strips of ileum were fixed in fresh $4 \%$ paraformaldehyde in $0.1 \mathrm{~mol} / \mathrm{L}$ phosphate buffered saline (PBS) at $4^{\circ} \mathrm{C}$ for 6 hours, then placed overnight in $20 \%$ sucrose in PBS at $4^{\circ} \mathrm{C}$. The specimens were coated in OCT embedding compound (Miles, Elkhart, IN), snap frozen in isopentanc/dry icc and storcd at $-70^{\circ} \mathrm{C}$. $10-\mu \mathrm{m}$ cryostat sections were mounted on $1 \%$ chrome-alum gel subbed slides, allowed to air dry, and placed in $0.1 \mathrm{~mol} / \mathrm{L}$ PBS for 5 minutes. Serial sections were subsequently incubated for 12 hours with a mouse monoclonal antibody raised against c-kit, (NCL-cKit, 57A5D8,
Novocastra Laboratories Ltd, Newcastle upon Tyne, UK; dilution, 1:50 with $0.1 \mathrm{~mol} / \mathrm{L}$ PBS), and a rabbit polyclonal antibody to Protein Gene Product 9.5, (PGP9.5; Biogenesis PLC; dilutivn, 1:2000 with $0.1 \mathrm{~mol} / \mathrm{L}$ PBS), a well characterised neuronal marker. ${ }^{18} \mathrm{~A}$ streptavidin $\mathrm{ABC}$ Duet kit (DAKO, Glostrup, Denmark) was subsequently used accordingly
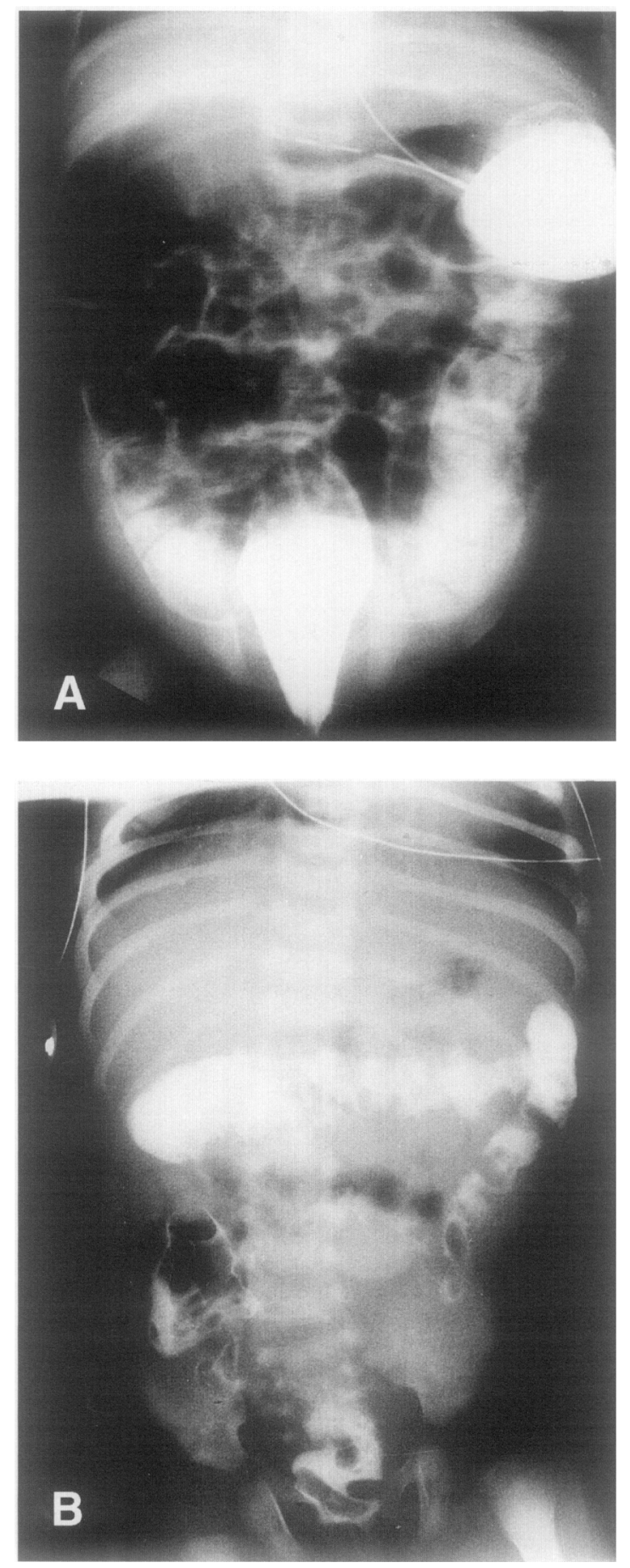

Fig 2. Case 2. (A) Enema with hydrosoluble contrast medium showing a normal rectum and absence of mechanical obstruction at the time of admission. (B) Plain abdominal radiograph obtained 10 days later showing persistence of contrast medium in the colon and rectum. 
with the manufacturers instructions to detect primary antibodies using 3,3-diaminobenzidine (DAKO) as the chromogenic substrate.

No staining was obscrved when the primary antibodics werc omitted. Mayer's H\&E-stained paraffin-fixed sections were also examined.

\section{Case 2}

Biopsy specimens of the muscular coats at various levels of the colon and small intestine obtained at the time of the two laparotomies were processed simultaneously as described previously. ${ }^{14,16}$ Briefly, the specimens were fixed overnight in fresh $4 \%$ paraformaldehyde solution in $0.1 \mathrm{~mol} / \mathrm{L}$ PBS at $4^{\circ} \mathrm{C}$, cryopreserved in graded solutions of sucrose (10\%, 20\%, 30\%; overnight each), properly oriented during embedding in Tissue-Tek OCT compound (Miles), snap-frozen in 2-methyl butane that had been cooled on dry ice, and stored at $-80^{\circ} \mathrm{C}$. Longitudinal sections ( $15 \mu \mathrm{m}$ thick) were cut on a cryostat, mounted on slides coated with $0.1 \%$ percent poly-L-lysine (Sigma, St Louis, MO) and stored at $-20^{\circ} \mathrm{C}$ until use. Immunohistochemistry testing was performed using commercially available kits using the avidin-biotin-complex (ABC) system (Vectastain@ABC). Different kits were used according to the species of the primary antibodies used. A rabbit polyclonal antiserum raised to a synthetic peptide corresponding to residues 961 to 976 of the human c-kit receptor (SC-39, Santa-Cruz Biotechnology, Santa-Cruz, CA; final dilution, 1:250) and mouse monoclonal antibodies raised against the neuron specific enolase (NSE; M873, DAKO; $5 \mathrm{mg} / \mathrm{mL}$ ) and the glial marker S-100 (SA2102, Affinity, Nottingham, UK; $1 \mathrm{mg} / \mathrm{mL}$ ) were used as described previously. ${ }^{14,16}$ Sections were first incubated in blocking normal serum for 20 minutes, then incubated with the primary antibody diluted in normal serum for 3 hours, rinsed in PBS for 10 minutes, before incubating with the appropriate secondary antibodies according to suppliers instructions (Vectastain@ $\mathrm{ABC}$, Vector, Burlingame, CA). Immunopure@ Metal-enhanced DAB substrate kit (Pierce, Rockford, IL) that produces a brown deposit was used as the chromogenic substrate. No staining was observed when the primary antibodies were onilled. Histochemical staining for NADPH-diaphorase, as a marker for the neuronal isoform of constitutive NO synthase, was performed as previously described. ${ }^{19}$ The slides were incubated in the dark in a solution of $0.1 \mathrm{~mol} / \mathrm{L}$ Tris $\mathrm{HCl}$ buffer containing $1 \mathrm{mmol} / \mathrm{L}$ NADPH (Sigma), $0.2 \mathrm{mmol} / \mathrm{L}$ Nitro Blue Tetrazolium (Sigma) and $10 \%$ dimethylsulfoxide at $37^{\circ} \mathrm{C}$ for 75 to 90 minutes. Omission of NADPH resulted in the absence of staining. In addition, sections were stained with Mayer's H\&E for routine evaluation

\section{RESULTS}

\section{Case 1}

H\&E preparations showed myenteric ganglion cells and normal muscle layers within the biopsy sections of terminal ileum. PGP9.5 staining confirmed the presence of normally distributed neuronal tissue. c-kit-positive ICC were completely absent from the first laparotomy specimen ( 31 weeks postconceptional age; Fig $3 A$ ). In the specimen obtained at the time of ileostomy closure, ICC were present in the myenteric plexus, circular and longitudinal muscle, and the inner aspect of circular muscle (42 weeks postconceptional age; Fig 3B).

\section{Case 2}

H\&E preparations showed myenteric ganglia with mature neurones and normal muscle layers in all the biopsy results. The distribution of NADPH-diaphorase activity and the distribution of neuronal (NSE immunoreactive) and glial (S-100 immunoreactive) elements was
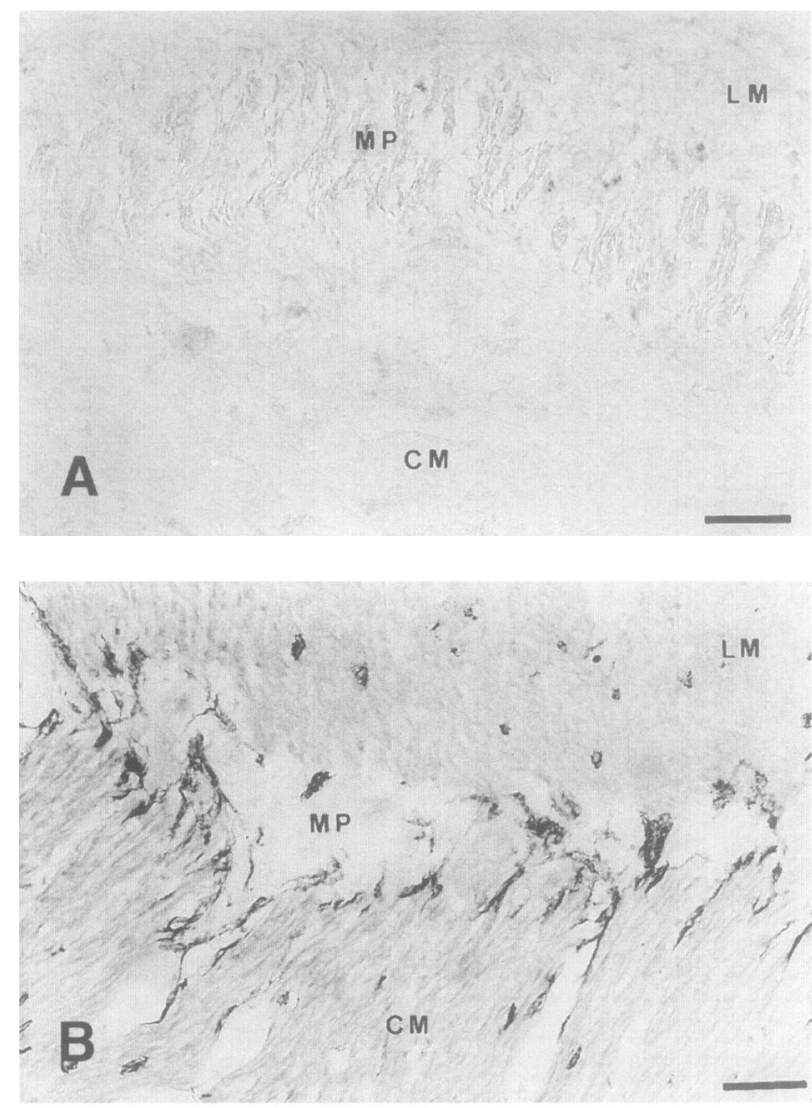

Fig 3. Case 1. Micrographs of sections of terminal ileum show (A) the absence of $\mathrm{c}$-kit immunoreactivity at 31 weeks' postconceptional age and (B) c-kit immunoreactive ICC surrounding the myenteric ganglia at the time of the second laparotomy ( 43 weeks' postconceptional age). CM, circular musculature; $L M_{r}$ longitudinal musculature; MP, myenteric plexus. (Scale bar, $\mathbf{5 0} \mu \mathrm{m}$.)

normal. The c-kit antiserum labelled normal-looking ICC in the myenteric plexus of the small intestine and of the colon. ICC were absent in the muscle layers and in the region of the submuscular plexus in the specimen of colon obtained at the time of the first laparotomy, when the colon was adynamic (Fig 4A). In contrast, in the second set of specimens obtained at the time colonic motility had spontaneously recovered, ICC were observed in the muscle layers and in the submuscular plexus (Fig 4B). The distribution of ICC in the terminal ileum from both operative specimens appeared normal (not shown).

\section{DISCUSSION}

To the best of our knowledge, the correlation of ICC development with gut motility in neonates with pseudoobstruction has not previously been reported. Neonates with idiopathic transient functional obstruction, also labelled meconium syndrome, have been described in the literature, but the aetiology of this disease has remained obscure. Infants with this syndrome are often premature and require prolonged parenteral nutrition, but have a good long-term outcomc in tcrms of cntcral function. ${ }^{4}$ 

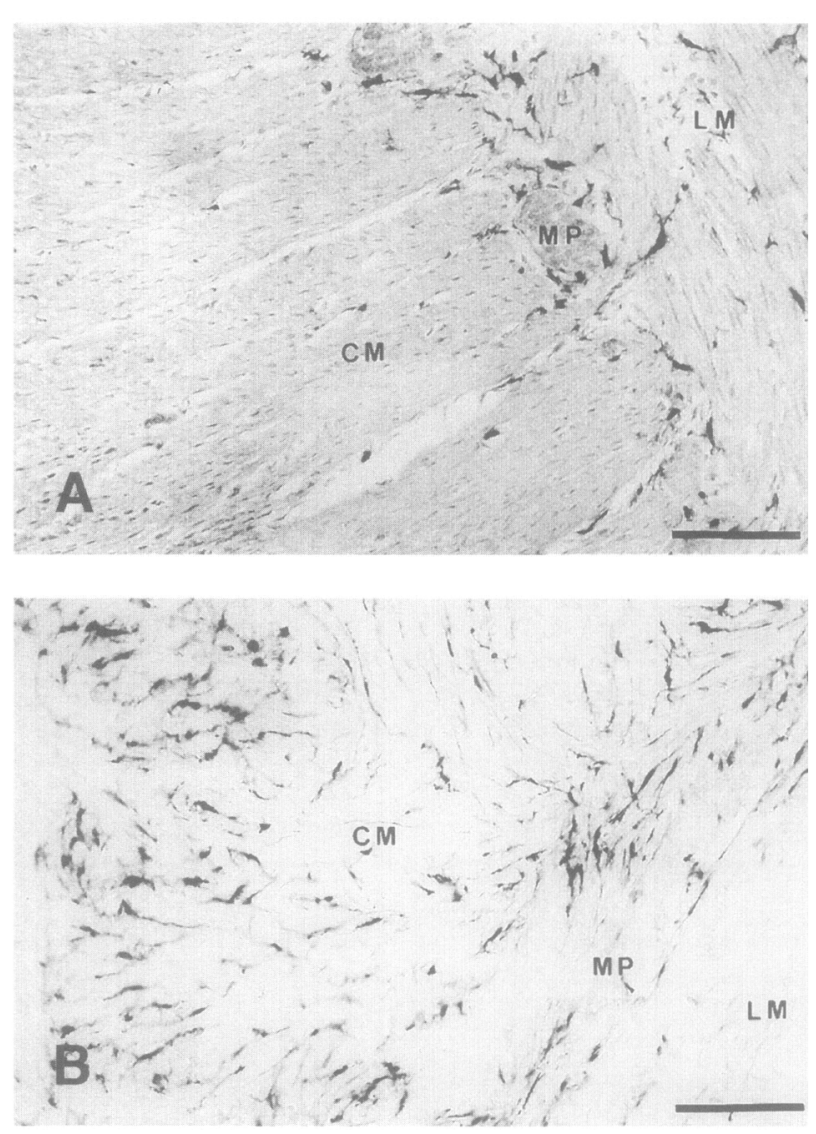

Fig 4. Case 2. Micrographs of sections of descending colon show (a) the absence of c-kit immunoreactivite ICC in the circular musculature at the time of first laparotomy (1 month of age) and (b) the presence of a normal pattern of c-kit immunoreactive ICC at the time of the second laparotomy (6 months of age). $\mathrm{CM}$, circular musculature; LM, longitudinal musculature; MP, myenteric plexus. (Scale bar, $100 \mu \mathrm{m}$.)

Our observations suggest that delayed maturation of ICC could be involved in the pathophysiology of the transient gastrointestinal dysmotility seen in such neonates in the absence of other identifiable causes. Lack of ICC has been associated with disturbances of gastrointestinal motility both in animal models ${ }^{9-11}$ and in human diseases. ${ }^{12-17}$ Therefore developmental delay of ICC may be implicated in neonatal pseudoobstructive syndromes. In case 1, (premature), ICC were initially absent, whereas in case 2 (at term), ICC were present in the myenteric plexus but absent from in the muscle layers and the submuscular plexus, perhaps reflecting different stages in ICC development.

Despite having been first identified over a century ago by the neuroanatomist Ramon y Cajal, ${ }^{20}$ it is only in recent years that the pivotal role of these mesenchymal cells in gut motility has been identified. The recent identification of the tyrosine kinase receptor c-kit has made the study of ICC easier. Studies in adult colon have showed a complex network of ICC incorporating the submucosal aspect of colonic circular muscle and the myenteric plexus. $^{21}$ No ontogenic study has been published about humans, and clearly this is of paramount importance in comparing putative cases of ICC developmental delay with normal human development. Immunohistochemical studies (Kenny et al 1997, submitted and unpublished personal observations) indicate a cephalocaudal gradient of development, with c-kit-positive ICC present in the antrum, distal small intestine, and caecum at 12 weeks postovulation in human embryos. By 26 weeks, well-developed ICC have been demonstrated in the myenteric plexus and submuscular plexus of terminal ileum of an infant with segmental ileal atresia. The time of appearance of ICC in the human distal fetal colon is not known but the ICC network is already developed in neonates. ${ }^{16}$

The interaction between ICC and the enteric nervous system is currently poorly understood. ICC are of mesenchymal origin and have been shown to develop independently of the enteric nervous system (ENS) in avian bowel. ${ }^{22}$ In human HD, the pattern of ICC in the aganglionic colon is abnormal, although ICC are present in reduced number, ${ }^{16}$ suggesting either that normal ICC development may be dependent on interactions with ganglion cells or that a common cause may produce both the defect in the enteric nervous system and failure in development of ICC as seen in HD. In the two cases reported here, the ENS appeared morphologically normal. However, developmental delay may be related to lack of trophic factors, neural or otherwise.

Developmental delay of ICC in the GI tract may be a new diagnosis to consider when assessing neonates with pseudoobstructive symptoms. Full attention should be given to eliminating all other possible causes of obstruction. Careful pathological evaluation of multiple surgical biopsy specimens is essential to support the diagnosis. The underlying physiopathologic mechanisms remains obscure. With adequate supportive treatment, including surgical intervention where indicated, the prognosis appears excellent. Spontaneous recovery within several weeks or months and a normal long-term GI function could be anticipated.

\section{REFERENCES}

1. Ricklanm PP, Bueckman CR: Neonatal meconium syndrome in the absence of mucoviscidosis. Am J Surg 106:173, 1965

2. Siegel MJ, Shackelford GD, McAlister WH: Nennatal meconium blockage in the ileum and proximal colon. Radiology 132:79-82, 1979

3. Vinograd I, Mogle P, Peleg O: Meconium disease in premature infants with very low weight. J Pediatr 103:963-966, 1983
4. Lamireau 'I', Millon A, Sarlangue J, et al: Le syndrome de pseudo-obstruction intestinale transitoire du premature. Arch FrPediatr 50:301-306, 1993

5. Thuneberg L, Rumessen JJ, Mikkelsen HB: Interstitial cells of Cajal-An intestinal impulse generation and conduction system? Scand J Gastroenterol (suppl) 71:143-144, 1982 
6. Thuneberg L: Interstitial cells of Cajal, in Schultz SG, Wood JD Rauner BB, (eds): Handbook of Physiology. v. 1. Bethesda, MD, American Physiological Society, 1989 pp 349-386

7. Sanders KM: A case for interstitial cells of Cajal as pacemakets and mediators of neurntransmission in the gastrointestinal tract. Gastroenterology 111:492-515, 1996

8. Maeda H, Yamagata A, Nishikawa S, et al: Requirement of c-kit for development of intestinal pacemaker system. Development 116:369375,1992

9. Ward SM, Burns AJ, Torihashi S, et al: Impaired development of interstitial cells and intestinal electrical rhythmnicity in steel mutants. Am J Physiol 269:C1577-1585, 1995

10. Huizinga JD, Thuneberg $\mathrm{L}$, Kluppel M, et al: W/kit gene required for interstitial cells of Cajal and for intestinal pacemaker activity. Nature 373:347-349, 1995

11. Isozaki $\mathrm{K}$, Hirota $\mathrm{S}$, Nakama $\mathrm{A}$, et al: Disturbed intestinal movement, bile reflux to the stomach, and deficiency of c-kitexpressing cells in Ws/Ws mutant rats. Gastroenterology 109:456-464, 1995

12. Yamataka A, Fujiwara T, Kato $Y$, et al: Lack of intestinal pacemaker (C-KIT-Positive) cells in infantile hypertrophic pyloric stenosis. JPS 31:96-99, 1995

13. Langer JC, Berezin I, Daniel EE: Hypertrophic pyloric stenosis: Ultrastructural abnormalities of enteric nerves and the interstitial cells of Cajal. JPS 30:1535-1543, 1995
14. Vanderwinden J-M, Liu H, De Laet M-H, et al: Study of the interstitial cells of Cajal in infantile hypertrophic pyloric stenosis. Gastroenterology 111:279-288, 1996

15. Yamataka A, Kato Y, Tibboel D, et al: A lack of intestinal pacemaker (c-kit) in aganglionic bowel of patients with Hirschsprung's disease. JPS 30:441-444, 1995

16. Vanderwinden JM, Rumessen JJ, Lin H, et al: Interstitial cells of Cajal in human colon and in Hirschsprung's disease. Gastroenterology 111:901-910, 1996

17. Yamataka A, Fujiwara T, Nishiye II, et al: Localization of intestinal pacemaker cells and synapses in the muscle layers of a patient with colonic hypoganglionosis. JPS 31:584-587, 1996

18. Wilson PO, Barber PC, Hamid QA, et al: The immunolocalization of protein gene product 9.5 using rabbit polyclonal and mouse monoclonal antibodies. Br J Exp Pathol 69:91-104, 1988

19. Vanderwinden JM, Mailleux P, Schiffmann SN, et al: Nitric oxide synthase activity in infantile hypertrophic pyloric stenosis. $\mathrm{N}$ Engl J Med 327:511-515, 1992

20. Cajal SR: Sur les ganglions et plexus nerveux de l'intestin. CR Soc Biol (Paris) 45:217-223, 1893

21. Rumessen JJ, Peters S, Thuneberg L: Light- and electron microscopical studies of interstitial cells of Cajal and muscle cells at the submucosal border of human colon. Lab Invest 68:481-495, 1993

22. Lecoin L, Gabella G, Le Douarin N: Origin of the c-kit-positive interstitial cells in the avian bowel. Development 122:725-733, 1996 